\title{
Sprachwissenschaftliche Analysen zur Kommunikation auf medizinischen Selbsthilfe-Plattformen - ein Beitrag zur Qualitätssicherung im interdisziplinären Kontext
}

\begin{abstract}
Die Interaktion zwischen medizinischen Laien auf Selbsthilfeplattformen ist mittlerweile als wichtiger Teil der Kommunikation über Gesundheit und Krankheit in der Gesellschaft zu verstehen. Es handelt sich dabei nicht um Fachkommunikation im engeren Sinne; fachliche Inhalte werden aber durchaus verhandelt, was beispielsweise Auswirkungen auf die weiteren Strategien Betroffener zur Bewältigung ihrer Krankheit und auch die Kommunikation mit Fachleuten haben kann. Ein interessantes Beispiel aus Sicht der Angewandten Linguistik ist dabei der Umgang von Laien mit fachlichen Kurzwörtern. Die folgenden Ausführungen sollen deshalb einen Beitrag sowohl zur Qualitätssicherung der Kommunikation auf Medizin-Foren ohne Expertinnen-Beteiligung leisten als auch - im späteren Verlauf zur pragmatischen Erforschung sprachlicher Kürze. ${ }^{1}$
\end{abstract}

Keywords: Wissenstransfer, Qualitätssicherung, Laien-Laien-Kommunikation, Selbsthilfeplattformen, Sprachliche Kürze

\section{1 „Der Patient im Netz“ - eine Hinführung aus linguistisch-funktionaler Sicht}

Die Rolle der Patient*innen hat sich mit den Möglichkeiten des Internets verändert. Die Betroffenen agieren tendenziell eigenständiger, was auch vom Gesundheitswesen verlangt wird (Adhärenz/Compliance): Nicht zuletzt der Kostendruck

1 Der Beitrag ist parallel zu meinen Überlegungen (aus anderer Perspektive) für eine Festschrift entstanden und wurde schließlich eine gekürzte und leicht geänderte Fassung dieses Festschriftbeitrags (Reimann, im Druck).

Sandra Reimann, Universität Oulu (Finnland), Germanistik, sandra.reimann@oulu.fi; Universität Regensburg (Deutschland), Institut für Germanistik, Deutsche Sprachwissenschaft, sandra.reim ann@ur.de

Ә Open Access. (C 2021 Sandra Reimann, published by De Gruyter. (cc) BY-NC-ND Dieses Werk ist lizenziert unter einer Creative Commons Namensnennung - Nicht-kommerziell - Keine Bearbeitung 4.0 International Lizenz. https://doi.org/10.1515/9783110688696-026 
macht eine effizientere Interaktion zwischen den Beteiligten notwendig (vgl. z. B. Thielscher \& Schulte-Sutrum 2016). Die Patient*innen sollen dabei gut informiert sein und sich bei gesundheitlichen Problemen aktiv und verantwortlich am Entscheidungsprozess hinsichtlich therapeutischer Maßnahmen beteiligen. Für die Patient*innen ist der Zugriff aufs Internet eine bedeutende Informationsquelle geworden (z. B. Diaz et al. 2002) ${ }^{2}$ - sie gehen heute gerne zuerst ins Netz und dann erst zum Arzt/zur Ärztin (z. B. Fritzen 2015$)^{3}$-, die somit auch im medizinischen Diskurs allgemein zu berücksichtigen ist (Busch \& Spranz-Fogasy 2015: 353). ${ }^{4}$

Dass es einen Bedarf am Austausch von Betroffenen bzw. medizinischen Laien untereinander gibt, zeigen Selbsthilfegruppen seit langem (u. a. zur Geschichte der Selbsthilfegruppen international, insbesondere bei Drogen- und Alkoholsucht, siehe Flora, Raftopoulos \& Pontikes 2010; zur Bedeutung von Selbsthilfegruppen international am Beispiel der USA - sowohl face to face als auch online - siehe Williams 2019).

Die Möglichkeiten der Informationsbeschaffung und des Austauschs unter Laien sind in den vergangenen Jahren durch digitale Angebote im Internet gestiegen (siehe z. B. auch Nickel et al. 2020): Erfahrungsberichte werden online gestellt, Bewertungen für Ärzte, Ärztinnen und Kliniken abgegeben und Selbsthilfe im Internet und in den Sozialen Medien in Anspruch genommen. ${ }^{5}$ Ein naheliegender Vorteil der Kommunikation ohne Ärzte und Ärztinnen ist beispielsweise der Austausch unter Gleichgesinnten, wie z. B. folgender Ausschnitt aus dem Beitrag (Posting) \#16 eines Threads ${ }^{6}$ des Prostatakrebsdiskussionsforums des Bundesverbandes Prostatakrebs Selbsthilfe e.V. (BPS \& KISP 2020b), um das es im Verlauf des Beitrags noch gehen wird, explizit zeigt: Leider haben die meisten im Bekanntenkreis wenig oder gar keine Betroffene mit denen sie sich austauschen können. Das erste Posting des Threads enthält Fragen, die den Wissenstransfer medizinischer

2 Quinn et al. (2013) weisen darauf hin, dass sich das Internet insbesondere bei Tumor-Erkrankungen in den letzten Jahren als primäre Quelle für die Suche und den Austausch von Informationen entwickelt hat.

3 Vgl. auch Breyer \& Burger (2014) (dort findet sich auch der Teiltitel dieses Kapitels). Siehe die Ausführungen zur zugehörigen Arbeitsgruppe in Kap. 2.

$4 \mathrm{Zu}$ Textsorten in der Medizin (heute) und dabei auch zur Bedeutung des Laien-Laien-Diskurses im Hinblick auf deren mögliche „Wirkung auf die Fachkommunikation“ und den „zunehmend dialogisch-orientierten Wissenstransfer“"vgl. Weinreich (2015: 401).

5 Exemplarisch zum Teilen von medizinischen Informationen in Sozialen Medien vgl. z. B. Moorhead et al. (2013) sowie Byron, Albury \& Evers (2013). Zu anderen digitalen Angeboten im Internet vgl. z. B. Johnson \& Ambrose (2006) über Online-Neo-Tribes zum Austausch über Multiple Sklerose und den Einsatz von „Storytelling“; zum Teilen von „Erzählungen“ über das Thema ,Geburt‘ in Online-Foren siehe Tienken (2013).

6 Aus Datenschutzgründen werden die Links der Posts nicht genannt, auch wenn der Zutritt zur Plattform und die Rezeption der Beiträge kein Passwort erfordern. 
Inhalte betreffen (z. B. Nun zu meinen Fragen: Auf dem Überweisungszettel des Urologen standen folgende Werte: PSA [Zahlen genannt; S. R.]; G3; Gleason [ ... Zahl genannt; S. R.] / Was haben diese Werte nun zu bedeuten? Natürlich habe ich schon ein bisschen gegoogelt), sich aber auch auf die Arzt-Patienten-Kommunikation und die Erfahrung anderer Betroffener beziehen (z. B. Was kommt nun auf meinen Vater zu?; ... von euren Erfahrungen berichten ... Mein Vater versteht von dem ganzen Fach-Chinesisch mit dem er bisher konfrontiert wurde, fast nichts.). Korrekturen der Antworten das kann hier nur angedeutet werden - erfolgen allerdings lediglich innerhalb der Gruppe der Kommunizierenden, zu denen (offiziell) keine Fachleute gehören.

Neben den Chancen der verschiedenen Online-Angebote für medizinische Laien hinsichtlich „Information, Interaktion und Therapie“ (Kleinke 2015: 413; Hervorhebung im Original) werden in der Literatur auch Risiken und Grenzen behandelt (siehe dazu Link 2019: 165-166, die einen Überblick zu Gesundheitsportalen und Online-Communitys gibt).

Aus der Perspektive der germanistischen Linguistik ist festzuhalten, dass die Kommunikation von medizinischen Laien untereinander im Internet noch nicht umfassend untersucht ist. Als Grundlage zur linguistischen Beschäftigung mit den Orten und Formen der Kommunikation medizinischer Laien im Netz kann beispielsweise die Zusammenschau zu Gesundheitsangeboten und -kommunikation im Netz von Rossmann und Stehr (2019) verstanden werden. Auch die Übersicht von Kleinke (2015) ist hier zu nennen: Sie geht u. a. auch auf die (teils moderierten) medizinischen Online-Selbsthilfegruppen (OSHGen) ein und erwähnt dabei Rollen (z. B. Expert Patients) und Funktionen (,(emotionales) Empowerment“) der Beteiligten neben den in der Literatur (s. z. B. oben Link 2019) bereits thematisierten Risiken ,virtueller und nicht-virtueller medizinischer Laienkommunikation (z. B. zu langes Verweilen im Laiensystem und Unterlassen von Vorsorgemaßnahmen vgl. Busch 1994; Potts 2005; Darcy \& Dooley 2007; Eysenbach u. a. 2004)“. Weiter gibt Kleinke (2015: 416-417) einen knappen Überblick über bisherige Forschungen zu Online-Selbsthilfegruppen, die auch die sprachwissenschaftliche Perspektive einschließen. $\mathrm{Zu}$ nennen sind ferner auch die Beiträge im Special Issue „Language and Health Online“ (Linguistik Online, 2017).

Die folgende Untersuchung soll einen Beitrag zu mehreren Bereichen der Angewandten Linguistik leisten: erstens allgemein zu Analysen der Kommunikation auf Medizin-Foren ohne Expert*innen-Beteiligung, welche bisher kaum untersucht wurde, ${ }^{7}$ zweitens zur Qualitätssicherung auf solchen Foren und drittens

7 Siehe die umfangreiche sprachwissenschaftliche Studie der Verfasserin zur Kommunikation auf der moderierten Selbsthilfeplattform hungrig-online.de für Menschen mit Essstörungen, die seitens der psychosomatischen Medizin mit der Motivation angeregt wurde, zu prüfen, ob die Kommunikation auf dieser Plattform sich positiv auf den Bewältigungsprozess der Krankheit 
zur pragmatischen Erforschung sprachlicher Kürze, welche beim Wissenstransfer eine Rolle spielt und über die Vermittlungsfunktion und die Frage nach der Verständlichkeit wiederum Auswirkungen auf die Qualität der Kommunikation hat. Dabei kann sich der Begriff der Qualität auf unterschiedliche Bereiche beziehen: Es kann z. B. die medizinisch-fachliche Korrektheit (soweit festlegbar) interessieren, die Hinzuziehung zuverlässiger Quellen (Transparenz) oder die Berücksichtigung der Netiquette ${ }^{8}$ der Plattform (wenn vorhanden). Grundsätzlich geht es darum, Einblicke zu bekommen, wie die Userinnen von der Kommunikation profitieren können.

\section{Programmatische Überlegungen zum Beitrag der Sprachwissenschaft bei der Qualitätssicherung medizinischer Selbsthilfeplattformen - am Beispiel der Plattform Prostatakrebs-Selbsthilfe e. V.}

Die folgenden Ausführungen sind im Kontext des Forschungsprojekts der interdisziplinären Regensburger Arbeitsgruppe „Der Patient im Netz“ (Medizin/Urologie, Medienwissenschaft, Medieninformatik und Informationswissenschaft, Sprachwissenschaft) zu sehen. Es wurde mit dem Ziel initiiert, die Qualität von Webseiten, Ärzteportalen, Selbsthilfegruppen, Diskussions- und Patientenforen zu Volkskrankheiten in Bezug auf die europäischen urologischen Leitlinien $\left(\mathrm{EAU}^{9}\right)$ und im Hinblick auf die Zielgruppe „Patienten/Laien” zu untersuchen und Handlungsempfehlungen zum Umgang mit diesen Angeboten zu verfassen (siehe dazu z. B. Bruendl et al. 2018). Es geht also einerseits um eine Qualitätssicherung

auswirken kann. Hintergrund ist ein grundsätzliches Bedürfnis seitens der psychosomatischen Medizin, die Wirksamkeit von Behandlungsmethoden und des Austauschs von Betroffenen im Internet nachzuweisen - vor allem im Hinblick und als Reaktion auf die therapeutische Unterversorgung und die steigende Zahl der von Essstörungen oder auch anderen (psychosomatischen) Krankheiten Betroffenen (Reimann 2018; siehe auch Reimann 2020, 2019 und 2012).

8 Siehe zur Netiquette z. B. früh Storrer \& Waldenberger (1998); außerdem seien Sharf (1999), Misoch (2006: 177-185), Kayany (2004), Strawbridge (2006), Bendel (2010) sowie Slivová \& Kozík (2014) erwähnt.

9 European Association of Urology: https://uroweb.org/guideline/prostate-cancer/ (letzter Zugriff am 27.03.2021). 
aus medizinisch-fachlicher Sicht, andererseits um eine zielgruppengerechte Kommunikation (gegenüber medizinischen Laien). ${ }^{10}$

Das Korpus stammt von den Seiten des Prostatakrebs-Diskussionsforums des Bundesverbands Prostatakrebs Selbsthilfe e. V. (BPS), einer Online-Selbsthilfeplattform für Prostatakrebs-Patienten. Im Forum sind 5.659 Benutzer^innen tätig, die seit 2004 131.965 Beiträge zu 11.318 Themen verfasst haben (Stand: 02.08.2021). ${ }^{11}$ Es gibt zwei offizielle Rollen: „Benutzerin“ (Betroffene, Angehörige) und „Moderator“. Diese Rolle wird derzeit nur von einer einzigen Person ausgeübt: Es handelt sich um einen selbst von der Krankheit Betroffenen, der medizinischer Laie ist und die Netiquette ${ }^{12}$ prüfen soll: Darin geht es ausschließlich um das kommunikativsozial angemessene Miteinander der Kommunikationsteilnehmer*innen (und nicht um die fachlich-medizinische Perspektive). ${ }^{13}$

Zum Vorgehen aus sprachwissenschaftlicher Sicht sind zunächst Vorüberlegungen anzustellen; vor allem ist zuerst zu fragen: Welchen Beitrag kann hier die Sprachwissenschaft überhaupt leisten? Es ist nämlich davon auszugehen, dass der Austausch über medizinische Sachverhalte thematisch bedingt - das Prostata-Karzinom ist „die häufigste Krebserkrankung des Mannes“14 - sicher einen hohen Stellenwert in der Kommunikation hat.

Festzuhalten ist: Zur Qualität der Kommunikation hinsichtlich medizinischer Korrektheit der behandelten Themen kann die Sprachwissenschaft im engeren Sinne nichts beitragen; stattdessen könnte beispielsweise ein Abgleich mit den medizinischen Leitlinien ${ }^{15}$ erfolgen und/oder die Kommentierung/Bewertung der

$10 \mathrm{Zu}$ „mangelnde[r] Qualität und Quellentransparenz“ vgl. auch Rossmann \& Stehr (2019: 409-412).

11 BPS (2020a), Webseite: https://www.prostatakrebs-bps.de/ (letzter Zugriff am 02.08.2021). BPS \& KISP (2020b), Webseite Prostatakrebs-Diskussionsforum: https://forum.prostatakrebsbps.de/ (letzter Zugriff am 02.08.2021); das Diskussionsforum wird von BPS und KISP, „Kontakt, Informations- und Selbsthilfestelle zum Prostatakrebs“, betrieben.

12 Siehe Bundesverband Prostatakrebs Selbsthilfe e. V. (2021): Forumsregeln: https://prostata krebs-bps.de/forum/forumsregeln/ (letzter Zugriff am 15.07.2021).

13 Weiterführende Anmerkungen zur Rollendifferenzierung und Funktion der beteiligten Akteure auf Selbsthilfeplattformen finden sich bei Reimann (2018), zur Prostata-Plattform speziell siehe Reimann (im Druck). Dort auch Näheres zum Wissenstransfer unter Laien und die in der Literatur schon ansatzweise diskutierten Konsequenzen für den Gesundheisdiskurs generell (siehe u. a. Weinreich (2015): 401).

14 Als Quelle kann z. B. Maximilian Burger, Direktor der Klinik für Urologie am Caritas-Krankenhaus St. Josef, Regensburg, genannt werden, hier: https://www.caritas-regensburg.de/aktu elles/presse/prostatakrebs-frueh-erkannt-gefahr-geba (letzter Zugriff am 25.09.2020).

15 Leitlinienprogramm Onkologie (2018): https://www.leitlinienprogramm-onkologie.de/fi leadmin/user_upload/Downloads/Leitlinien/Prostata_5_0/LL_Prostata_Langversion_5.0. pdf (letzter Zugriff am 14.04.2020). 
für die Untersuchung herangezogenen Forentexte durch medizinische Expertinnen, um beispielsweise folgende Fragen zu beantworten: Welche Informationen sind falsch oder unvollständig? Wie viele Informationen gehen auf dem Weg von Patient A zu Patient B verloren? Für eine erste medizinische Bewertung ausgewählter Beispiele zum Umgang mit Kurzwörtern wurde der Urologe Johannes Breyer aus der Arbeitsgruppe „Der Patient im Netz“ hinzugezogen. ${ }^{16}$

Die Rolle der Sprachwissenschaft dürfte zunächst sein, über die Kommunikation zu reflektieren, schließlich Mittlerin und Moderatorin gegenüber der Medizin, die die fachliche Bewertung vornehmen kann, zu sein und abschließend Handlungsempfehlungen für die Verbesserung der Kommunikation zu geben.

Sprachwissenschaftlichen Untersuchungen zugrundeliegende Fragen auf einer solchen Medizin-Plattform könnten dabei folgende sein:

- Wer sind die an der Kommunikation Beteiligten?

- Welche Themen werden verhandelt (z. B. zu Diagnostik, Therapie und Prävention, zwischenmenschlichen Erfahrungen) und wie werden sie im Austausch versprachlicht (z. B. subjektiv/emotional)?

- Wie wird medizinischer Fachwortschatz (Fachwörter, Kurzwörter) eingesetzt (z. B. hinsichtlich der Verständlichkeit)?

- Welche Mechanismen der Reflexion finden sich?

- Auf welche Weise wird evtl. intramediale (Sprach-)Kritik der Beteiligten (Benutzer^in, Moderator) ausgeübt?

- Welche Funktionen der Plattform zeigen sich über die Postings?

Im Folgenden erfolgt eine Fokussierung auf sprachliche Kürze ${ }^{17}$ und dabei auf den Kurzwortgebrauch im Rahmen der vorliegenden Laien-Laien-Kommunikation, weil hier explizit der Wissenstransfer - in dem Fall unter Laien - eine Rolle spielt: Wird er überhaupt vorgenommen? Wenn das der Fall ist, werden folgende Fragen relevant: Wie wird der Wissenstransfer durchgeführt (z. B. Einsatz von Vollformen und somit Auflösen der Kurzwörter, Beispiele, Übersetzungen, Anführen von Quellen,

16 Die Befragung hat Bettina Dums (Universität Regensburg) durchgeführt. Alle Anmerkungen des Urologen Johannes Breyer zum Wissenstransfer stammen aus einem Gespräch bzw. schriftlichen Kommentaren vom 19.03.2019 (siehe Dums (2020)). Die darauf aufbauenden Untersuchungen von Bettina Dums (2021) zur P(-Selbsthilfe-Plattform gehen in den vorliegenden Beitrag ein.

17 In jüngerer Zeit wird dabei verstärkt zur Theorie kurzer Texte geforscht, z. B. Hausendorf (2009), Janich (2015), Leyhausen (2007), Lorenz \& Nehrlich (2019), Nikula (2015) und Pappert \& Roth (2021). Siever (2011) behandelt alle möglichen Aspekte der Kürze aus linguistischer Sicht. 
Verweise, Formen des Erklärens, weiterführende Informationen für den Laien)? ${ }^{18}$ Welche Formen finden sich (wie häufig)? Welche Bedeutungen werden den Kurzwörtern zugeschrieben? Vor allem bei den fachsprachlichen Kurzwörtern stellt sich zunächst die Frage der inhaltlich angemessenen Dekodierung durch die medizinischen Laien. Lässt sich aus der Kommunikation erkennen, mit welcher Bedeutung das Kurzwort im vorliegenden Kontext verwendet wird? Werden Schwierigkeiten bei der Entschlüsselung kommuniziert? Wenn ja: Wie wird darauf reagiert? Werden mögliche Verstehensprobleme vorweggenommen und wird ein Wissenstransfer angebahnt? In der folgenden Analyse geht es um den zum Zeitpunkt der Analyse 70 Beiträge umfassenden Thread „Neu hier, Bitte um Informationen“, ${ }^{19}$ da hier schon aufgrund des Titels Wissenstransfer zu erwarten ist.

\section{Wissenstransfer, Fachlichkeit und sprachliche Kürze - Analysen zum Kurzwortgebrauch im Thread „Neu hier, Bitte um Informationen“}

Hauptmerkmal des Kurzworts ist eine Veränderung der Ausdrucksseite im Vergleich zur zugehörigen Vollform; eine inhaltliche Modifikation liegt in der Regel nicht vor. Kurzwörter finden sich in 37 der insgesamt 70 Beiträge zum vorliegenden Thread; insgesamt werden Kurzwörter 105-mal zum Einsatz gebracht, wobei 35 verschiedene Kurzwörter verwendet werden. An diesem Thread sind 22 User aktiv beteiligt. Acht dieser User schreiben nur einen einzigen Beitrag. Die meisten Beiträge, die ein User verfasst, sind elf. Die meisten Kurzwörter (je sieben) kommen in den Beiträgen \#1 (Eröffnungsbeitrag der Fragestellerin mit unmarkierten Zitaten von auf einer Überweisung aufgeführten Kurzwörtern) und \#50 (ein Beitrag des Moderators) vor. ${ }^{20}$

18 Beispiele und Formen des Erklärens im medizinischen Kontext finden sich beispielsweise bei Brünner (2011), Wiese (2002) und Schuldt (1998), zu weiteren Themen siehe auch ThimMabrey \& Rössler (2020). Wegen der Vergleichbarkeit hinsichtlich zielgruppenspezifischer Gestaltung sind auch die Leitlinien (für medizinische Expert`innen) und Patienteninformationen (für Laien) zu verschiedenen Krankheiten interessant. Sie finden sich bei AWMF online: https://www.awmf.org/leitlinien.html (letzter Zugriff am 27.03.2021).

19 Thread Neu hier, Bitte um Informationen: https://forum.prostatakrebs-bps.de/showthread. php?10690-Neu-hier-Bitte-um-Informationen (letzter Zugriff am 21.04.2020).

20 Die folgend genannte erste Zahl steht für den Beitrag, die zweite für die Stelle, an der das Kurzwort in der „Kurzwortreihe“ dieses Beitrags steht (z. B. 50-6 BPS: Beitrag 50, sechstes Kurzwort in diesem Beitrag). 
Am häufigsten lassen sich die Kurzwörter PSA (Ersterwähnung: 01-1 PSA-Wert) und $P K$ (12-1) nachweisen - nämlich je elf Mal. Zum ersten Beispiel findet sich nie die Vollform Prostataspezifisches Antigen. ${ }^{21}$ Im zweiten Fall liegt die Vollform Prostatakrebs neun Mal in sieben Beiträgen vor (\#1, \#2 (2-mal), \#4, \#10, \#48, \#50 (2-mal), \#53).

Bei den Vollformen finden sich besonders häufig jene zu Uro (Ersterwähnung in Beitrag \#7: Uro-Onkologen, ein Kompositum mit unisegmentalem Kurzwort (Kopfwort)); eine Vollform gibt es 33-mal - in den Beiträgen \#1 (3-mal), \#3, \#6 (3), \#7, \#8 (3), \#9 (3), \#11, \#12 (6), \#13, \#16 (2), \#19 (4), \#32 (2), \#53, \#58 und \#60. Die Vollformen lauten: Urologe(n) (29-mal), Urologie (2-mal) und Urologie-Studium (2-mal).

Insgesamt handelt es sich überwiegend um Kurzwörter mit fachsprachlicher Bedeutung bzw. entsprechendem Zusammenhang oder Hintergrund. Nicht der medizinischen Fachsprache zugeordnet werden nur wenige Beispiele wie PDF-Datei, Docs und TE (= Threadersteller ${ }^{\star}$ in).

Klassifiziert nach Kurzwortbildungstypen kommen multisegmentale Kurzwörter (siehe etwa Fleischer \& Barz 2012), ${ }^{22}$ z. B. NW aus zwei Segmenten und COPD aus vier Segmenten, fast doppelt so häufig vor wie unisegmentale Kurzwörter (z. B. die Kopfwörter Gleason, Szinti).

Lediglich zu elf Kurzwörtern, also etwa einem Drittel, gibt es - an möglicherweise irgendeiner Stelle im Thread - auch Vollformen. Das ist eher wenig vor dem Hintergrund des Wissenstransfers. Zudem werden sie in den seltensten Fällen dazu unten mehr - in Bezug zur Kurzform gebracht. Ansonsten treten beispielsweise Voll- und Kurzform in unterschiedlichen Beiträgen auf. Andererseits kann man ein gemeinsames Vorwissen der User auf dieser Plattform annehmen, das nicht zuletzt auf den vom Moderator der Plattform verfassten Text „Erster Rat“ (Basiswissen“) $)^{23}$ zurückzuführen sein dürfte: Dieser Text wird beispielsweise im hier

21 Siehe z. B. die Erklärung in der sog. Patientenleitlinie „Früherkennung von Prostatakrebs Information für Männer“ im Leitlinienprogramm Onkologie (2015): Patientenleitlinie_Prostatakrebs_Frueherkennung_2015.pdf (letzter Zugriff am 27.03.2021).

22 Zur Kurzwortforschung exemplarisch z. B. Balnat (2011), Greule (2007), Kobler-Trill (1994).

23 Das aus dem Forum herunterladbare Dokument „Erster Rat.pdf“ (Damm 2020) wird von den Usern umgangssprachlich als „Basiswissen“ bezeichnet. Auf 209 Seiten hat der Moderator des Forums eine Einführung in das Thema Prostatakrebs erstellt, die einen zentralen Informationstext für die Nutzergemeinschaft des Forums darstellt. Aus der Perspektive des Wissenstransfers und der Qualitätssicherung - darauf wird folgend nicht weiter eingegangen - ist dabei positiv anzumerken, dass der Moderator - ein medizinischer Laie - das Dokument ständig verbessert und regelmäßig überarbeitet (zuletzt am 31.03.2020, Stand: 30.04.2020). Download-Link: http:// www.prostatakrebse.de/informationen/pdf/Erster\%20Rat.pdf (letzter Zugriff am 30.04.2020). Für die Analyse wurde die Version vom 31.03.2019 benutzt, die auf medizinische Korrektheit von Johannes Breyer (Urologe und Mitglied der Arbeitsgruppe „Der Patient im Netz“) überprüft und 
untersuchten Thread bereits zum ersten Mal in Beitrag 2 - also als Antwort auf den Eröffnungsbeitrag - genannt (Oben auf dieser Seite gibt es einen gelben Link der auf „Basiswissen“ verweist. Dieses Buch kann viele Deiner Fragen beantworten.). ${ }^{24}$ Die Beispiele, zu denen sich Vollformen finden, lauten: Gleason (9/2 Gleason Score ${ }^{25}$; hinzu kommt ein Mal die Kurzform GS 8), Uniklinik (2/1 Universitätsklinikum), Uro-Onkologen (4/33; zu Uro finden sich die Vollformen Urologe, Urologie-Studium), PCa-Geschichte (6/3 Prostatakarzinom ${ }^{26}$ : Die Vollform bezieht sich also nur auf das Kurzwort), Szinti (1/10; folgende Vollformen treten auf: Knochenszintigraphie, Skelettszintigraphie, Szintigraphie, Szintigramm ${ }^{27}$ ), PK (11/9; 8-mal Prostatakrebs, 1-mal Prostatakrebszentrum), OP (9/2 Operation), RPE (3/3 Prostatektomie $\left.{ }^{28}\right)$, Da Vinci (2/1 da-Vinci-System), Prostata (1/9; Vollform wie bei $P K)$ und NW (1/2 Nebenwirkung(en)). Vollformen zu Uro, Szinti und $P K$ bzw. Prostata treten also am häufigsten auf. Das zuletzt genannte Kurzwort - einmaliges Auftreten in Beitrag \#31 - dürfte als umgangssprachliche Variante mit intertextuellem Bezug interpretierbar sein, weil es strukturell an die populäre ${ }^{29}$ Konstruktion „Ich habe Rücken“ (nach Hape Kerkelings Figur „Horst Schlämmer“) erinnert:

insgesamt recht positiv beurteilt wurde. Neben diesem Dokument gibt es auch ein Abkürzungsverzeichnis, das ebenfalls vom Moderator erstellt wurde. Download-Link: http://prostatakrebse. de/informationen/pdf/abkuerzungen.pdf (letzter Zugriff am 24.07.2021). Probleme mit der Auffindbarkeit dieser Texte werden auch in den Postings thematisiert (siehe u. a. Dums 2021: 56).

24 Überhaupt werden die Möglichkeiten der Hypertextualität und des intertextuellen Verweisens (auch auf andere Online-Angebote) in diesem Thread gerne genutzt, was interessant sein dürfte mit Blick auf digitales User-Schreiben im Zusammenhang mit Wissenstransfer.

25 „9/2“ bedeutet, dass neun Mal die Kurzform und zwei Mal die/eine Vollform auftritt. Im Anschluss an die Zahlenkombination wird dann die Vollform genannt.

26 Die Vollform Prostatakarzinom zum Kurzwort $P C a$ wird in der oben erwähnten Leitlinie in Kap. „1.10. Verwendete Abkürzungen“ (S. 15) genannt. Das Kurzwort dürfte auf das englischsprachige Fachwort prostate cancer zurückzuführen sein.

27 Johannes Breyer vermerkt zum Kurzwort aus fachsprachlicher Sicht: „Szinti ist eine umgangssprachliche Abkürzung für Szintigraphie. Die Szintigraphie bezeichnet eine Untersuchungsmodalität ähnlich eines CT oder MRT. Daher ist es im Einzelfall wichtig zu spezifizieren, um was genau es sich handelt: Skelettszintigraphie oder Schilddrüsenszintigraphie etc. Im Falle des Prostatakarzinoms handelt es sich immer um eine Skelettszintigraphie (Knochenszintigraphie würde sinngemäß das Gleiche bedeuten, ist allerdings auch eher umgangssprachlich)“.

28 Das Adjektiv radikale (für „R“) fehlt in allen drei Erwähnungen, die ausschließlich in Beitrag \#19 - also nicht im Beitrag, in dem das Kurzwort vorkommt - zu finden sind.

29 Zum Beispiel Personalfitness (2017): https://www.personalfitness.de/lifestyle/292 (23.05.2017) oder Gala (2007): https://www.gala.de/stars/news/hape-kerkeling-ich-habe-ru ecken-20006850.html (letzter Zugriff jeweils am 01.10.2020). 
Wenn ich in meinem mUnfeld schaue dann kenne ich schon so viele die die selbe blöde Krankheit Prostata mit und ohne Op durchmachen.

Es handelt sich dabei - wie angesprochen - um ein Kurzwort zur Vollform Prostatakrebs, welche sich acht Mal findet. Thematisch passen dazu die weiteren (inhaltlich synonymen) Benennungen PK (Prostatakrebs), PCa und Pca (Prostatakarzinom).

Die Kurzwörter sind häufig Teil von Komposita, allerdings fehlen bei komplexen Formen oftmals die grammatisch/orthographisch notwendigen Bindestriche zwischen den unmittelbaren Konstituenten: PCa-Geschichte, PSA-Wert (auch PSA Wert), DKG zertifiziertes Zentrum, PI RADS 2, PSMA-PET/CT (auch PSMA PET/CT), Abdomen MRT, Uniklinik, Uro-Onkologen, PK-Rezidiv, PK-Zentren.

Dabei gibt es zu einigen der genannten Kurzwörter in Komposita keine Vollformen. Das gilt für folgende: PSA-Wert (11-mal; PSA=Prostataspezifisches Antigen), Abdomen MRT (9-mal; MRT = Magnetresonanztomografie), PSMA-PET/CT (3-mal; $P S M A=$ Prostataspezifisches Membranantigen, $P E T=$ Positronen-Emissions-Tomografie, $C T=$ Computertomografie), DKG zertifiziertes Zentrum (1-mal; DKG = Deutsche Krebsgesellschaft) und PI RADS 2 (1-mal; Prostata Imaging - Reporting and Data System Version 2). ${ }^{30}$

Im Hinblick auf den Umgang mit den verwendeten Kurzwörtern lässt sich nur in fünf Fällen in den insgesamt 70 Beiträgen von einer Art Wissenstransfer sprechen. Das betrifft die Kurzwörter ADT, Gleason, RPE, Da Vinci [Da-Vinci] und mpMRT. Alle anderen vorkommenden Kurzformen werden nicht in Bezug zur Vollform gebracht.

Das Kurzwort $A D T$ - ein multisegmentales Buchstabenkurzwort aus drei Segmenten - kommt nur einmal vor, und zwar in Beitrag \#3, also sehr früh in diesem Thread und fast unmittelbar nach dem Eröffnungsposting: Bei der berücksichtigung des Alters, und dem Ergebnis von Glasson 8, wird von den Urologen oft eine ADT (Anti-Hormon-Behandlung) empfohlen. Der Wissenstransfer durch den medizinischen Laien erfolgt durch eine Erklärung in Klammern: ADT (Anti-Hormon-Behandlung). Der User geht also davon aus, dass die Fragestellerin noch über wenig Vorwissen verfügt.

Das unisegmentale Kurzwort (Kopfwort) Gleason findet sich erstmals im Eröffnungsposting: Auf dem Überweisungszettel des Urologen standen folgende Werte: PSA [Zahlen genannt; S. R.]; G3; Gleason 8 / Was haben diese Werte zu

30 Die meisten Vollformen wurden über das medizinische Nachschlagewerk Pschyrembel (https://www.pschyrembel.de/) ermittelt, die Vollform zu DKG stammt von der Seite https:// www.krebsgesellschaft.de/ (letzter Zugriff am 01.09.2020). Zu PI RADS 2 siehe z. B. https://ra diopaedia.org/articles/prostate-imaging-reporting-and-data-system-pi-rads-1 (letzter Zugriff am 01.10.2020). 
bedeuten? Insgesamt kommt es acht Mal vor, die Vollform findet sich zwei Mal (Beiträge \#5 und \#19). Eine Erklärung wird bei der zweiten Erwähnung vorgenommen. Der Verfasser geht direkt und gleich zu Beginn seines Beitrags auf die Fragestellerin ein: Gleason Score bildet die Aggressivität des Tumors ab. Als Basis $6(3+3)$ Niedrigrisiko bis $10(5+5)$ Hochrisiko.

Das Kurzwort RPE - ein multisegmentales Buchstabenkurzwort aus drei Segmenten - kommt drei Mal vor, und zwar immer in Beitrag \#17. Der Wissenstransfer erfolgt über den Versuch einer Erklärung quasi durch vergleichende Negation und den Verweis auf Beispiele:

Die ist - ob roboter-assistiert oder minimal invasiv oder offen - kein Eingriff wie Blinddarm oder einen Stent setzen. Du kannst dazu hier oder auf myprostate.eu Fallbeispiele lesen und deinem Vater nahebringen.

Das unisegmentale Kurzwort (Kopfwort) Da Vinci [Da-Vinci] findet sich zwei Mal (Beiträge \#18, \#24), die Vollform da-Vinci-System ein Mal (Beitrag \#19). Dort - im Kontext der Vollform - erfolgt der Verweis auf den vom Moderator - und medizinischen Laien - verfassten Text „Basiswissen“ auf der Prostata-Plattform: Göttingen bietet auch die schonende Variante mit dem da-Vinci-System an (Abschnitt 8.1.4 im „Basiswissen“).

Das Kurzwort mpMRT - ein multisegmentales Buchstabenkurzwort aus fünf Segmenten - kommt erstmals in Beitrag \#5 vor (insgesamt sieben Mal: Beiträge \#5, \#47 (2-mal), \#50 (2-mal), \#53 (2-mal)); eine Vollform - sie lautet multiparametrische Magnetresonanztomographie ${ }^{31}$ - ist in keinem Beitrag vorhanden. Bei der letzten Nennung in Beitrag \#53 erfolgt ein Wissenstransfer mittels der Erläuterung von Folgen und Optionen verschiedener Diagnosemöglichkeiten inklusive persönlicher Überlegungen des Users:

Die einmalige systemische Sextantenbiopsie ist alleine nicht besser als ein mpMRT nach PI RADS 2. Letzteres ermöglicht zusätzlich eine gute Prognose auf extrakapsuläre Ausbreitung und Samenblaseninfiltration. Ferner ist es Voraussetzung für eine Fusionsbiopsie, und diese wiederum erlaubt zuverlässiger, AS oder fokale Behandlungen einzusetzen. Warum das nicht in die Leitlinie Einlaß findet, weiß ich nicht. Kosten/Nutzen kann es nicht sein. Und natürlich würden den Urologen Hunderttausende von Biopsien entzogen - dafür müßte man ihnen (als guter Verhandler) in der Leitlinie vielleicht ein Screening schenken, schließlich haben irgendwann fast alle ein (häufig erstmal kleines) PCa.

31 Prostata Hilfe Deutschland [sic!], Ausführungen des Urologen Frank Schiefelbein zum Terminus mpMRT: https://www.prostata-hilfe-deutschland.de/prostata-news/multiparametri sche-mrt-mpmrt (letzter Zugriff am 27.03.2021). 


\section{Zur Qualitätssicherung - Bewertung und Handlungsempfehlungen der Kommunikation auf Selbsthilfeplattformen}

Der medizinisch-fachlich korrekte Umgang mit den Kurzwörtern ADT, Gleason, $R P E, D a$ Vinci [Da-Vinci] und mpMRT, zu denen Strategien des Wissenstransfers durch die User der Plattform ausfindig gemacht werden konnten, kann aus sprachwissenschaftlicher Sicht nicht beurteilt werden. Die Ausführungen der User wurden daher einem Mediziner der interdisziplinären Regensburger Arbeitsgruppe „Der Patient im Netz“ zur Bewertung vorgelegt, um mittels des exemplarisch untersuchten Materials und der Beschäftigung mit Kurzwörtern einen ersten Eindruck davon zu bekommen, ob Handlungsbedarf hinsichtlich der Qualitätssicherung besteht. Als korrekt stuft der Urologe Johannes Breyer die Erklärung zum Gleason Score ein, die er lediglich dahingehend ergänzt, dass das „Wachstumsmuster des Tumors“ beschrieben und „hiermit eine Aussage über die Aggressivität des Tumors“ gegeben wird. Auch die Aussagen zum Da-Vinci-System ergänzt der Experte lediglich (die Ausführungen im „Basiswissen“ stuft er als korrekt ein): „Die robotisch-assistierte Operation kann man als schonender bezeichnen. Die Patienten sind schneller auf den Beinen, haben weniger Schmerzen, kleinere Wunden und es sind weniger Bluttransfusionen erforderlich“; weiter sei dieses Fachwort der „Eigenname des Operationsroboters“. Die Erklärungen zum Kurzwort ADT (Anti-Hormon-Behandlung) werden korrigiert: „Eigentlich Hormonentzugsbehandlung“. ${ }^{32}$ Bei zwei Beispielen übt der Mediziner Kritik am vorgenommen Wissenstransfer durch Laien. Die Ausführungen zum Kurzwort RPE findet er „sehr unspezifisch und laienhaft. Operative radikale Entfernung von Prostata und Samenblasen.“ Die Kommunikation zum Kurzwort zur mpMRT bezeichnet der medizinische Experte teils als „korrekt“, „nicht korrekt“ und „tendenziös“. Ergänzt sei noch, dass das oben erwähnte Kurzwort $P K$ für Prostatakrebs - laut Johannes Breyer - „fachsprachlich so nicht üblich“ sei.

Resümierend kann festgehalten werden, dass in den 70 Beitragen des ausgewählten Threads eine breite Palette an Kurzwörtern zu finden ist, die im Rahmen der vorliegenden Laien-Laien-Kommunikation (aus der Perspektive des Fachs Medizin) zum Einsatz kamen. Die meisten Kurzwörter gehören in den

32 Die ermittelte Vollform zu ADT lautet „Androgendeprivationstherapie“ (Takeda Pharma Vertriebs GmbH \& Co. KG, prostata.de: Eintrag „ADT“: https://www.prostata.de/lexikon/adt. Letzter Zugriff am 08.07.2020). 
fachlichen Kontext. Selten erfolgt dabei ein Wissenstransfer von Laie zu Laie. Mutmaßlich wird von gemeinsamem Vorwissen und von der Lektüre des vom Moderator erstellten Texts „Erster Rat (Basiswissen)“, auf den im vorliegenden Thread bereits in Beitrag 2 hingewiesen wird, ausgegangen oder nicht über Verständnisprobleme reflektiert. Dass der unbegleitete Austausch fachlicher Themen Falschinformationen übermitteln kann, wird durch die Kommentare des hinzugezogenen Urologen Johannes Breyer deutlich. So wäre zu überlegen, wie mit diesem Ergebnis umzugehen ist: Man könnte beispielsweise Fachleute einsetzen, die die Kommunikation auf der Laien-Laien-Plattform - zumindest zeitweise - betreuen, bewerten und gegebenenfalls korrigieren. Auch ein gut sichtbarer Hinweis auf die Rollen und Funktionen der Beteiligten auf der Plattform könnte zur Qualitätssicherung beitragen. Die Linguistik kann den medizinischen Fachleuten Hinweise auf „Stolpersteine“ beim Wissenstransfer durch Laien geben (z. B. bieten sich Analysen zum Umgang mit Fachwortschatz (über den Kurzwortgebrauch hinaus) durch medizinische Expert`innen an). Man könnte auch Transparenz hinsichtlich der Quellen bei der Heranziehung medizinischer Informationen einfordern. Aus der Perspektive der Qualitätssicherung ist nämlich weiter festzuhalten, dass auf der Prostatakrebs-Plattform - nach den bisherigen exemplarischen Untersuchungen auch über den Kurzwortgebrauch hinaus - die Kommunikation über medizinisch-fachliches Wissen (Therapien usw.) dominiert. Dabei ist Vorsicht bei der Rezeption dieser (medizinisch-fachlichen) Themen geboten, da in der Regel nur medizinische Laien kommunizieren: Es besteht das Problem medizinischer Korrektheit, fehlender Korrektur der Beiträge und vermutlich der selektiven Wahrnehmung durch die User, die in der Regel als Betroffene auch aus ihrer persönlichen Perspektive kommunizieren; ${ }^{33}$ dabei geht es auch um das Klammern an jeden Strohhalm bei schweren Krankheiten. Mit Blick auf die beteiligten Akteure in Online-Foren wie der Prostataplattform - medizinische Expert*innen sind (offiziell) nicht dabei - ist daher das Einholen einer Zweitmeinung anzuraten: Für die Informationsfunktion im engeren Sinne (als Informationsbeschaffung) wäre es wohl besser, andere Ratgeber-Plattformen zu nutzen, z. B. Onmeda (Online-Diskussion teilweise noch mit Expert^innenrat: Beteiligung von Mediziner*innen als Ratgeber*innen), ${ }^{34}$ oder den Weg in eine Arztpraxis auf sich zu nehmen.

33 Albert Busch (1999: 105) beispielsweise weist darauf hin, dass medizinisches Laienwissen bzw. „gemeinsprachliche Bedeutungen“ zu Gesundheit und Krankheit u. a. geprägt sind „von nichtfachlichen Stereotypen (Vorwissen)“, „,von der (intertextuellen) Einbindung in Diskurse“ und ,vom Medium“.

34 Onmeda.de (letzter Zugriff 09.07.2021). 


\section{Literatur}

\section{Forschungsliteratur}

Balnat, Vincent (2011): Kurzwortbildung im Gegenwartsdeutschen. Hildesheim, Zürich, New York: Olms.

Bendel, Oliver (2010): Netiquette 2.0 - der Knigge für das Internet. Netzwoche 5, 40-41.

Breyer, Johannes \& Maximilian Burger (2014): Der Patient im Netz - Chance oder Risiko? Mitteilungen des Regensburger Verbunds für Werbeforschung (RVW). Online-Magazin 2 (2014), 33-35. http://epub.uni-regensburg.de/rvw.html/ (letzter Zugriff 09.07.2021).

Bruendl, Johannes, Clemens Rothbauer, Bernd Ludwig, Bernhard Dotzler, Christian Wolff, Sandra Reimann, Hendrik Borgmann, Maximilian Burger \& Johannes Breyer (2018): Accordance of Online Health Information on Prostate Cancer with the European Association of Urology Guidelines. Urologia Internationalis 100 (3), 288-293.

Brünner, Gisela (2011): Gesundheit durchs Fernsehen - Linguistische Untersuchungen zur Vermittlung medizinischen Wissens und Aufklärung in Gesundheitssendungen. Duisburg: Universitätsverlag Rhein-Ruhr.

Busch, Albert (1999): Semantische Vertikalitätstypik und diskursive Grundkonzepte in der Gesundheitskommunikation. In Kirsten Adamzik \& Jürg Niederhauser (Hrsg.), Wissenschaftssprache und Umgangssprache im Kontakt (Germanistische Arbeiten zu Sprache und Kulturgeschichte 38), 103-122. Frankfurt a. M. u. a.: Peter Lang.

Busch, Albert (1994): Laienkommunikation. Vertikalitätsuntersuchungen zu medizinischen Experten-Laien-Kommunikationen. Frankfurt a. M. u. a.: Peter Lang.

Busch, Albert \& Thomas Spranz-Fogasy (2015): Sprache in der Medizin. In Ekkehard Felder \& Andreas Gardt (Hrsg.), Handbuch Sprache und Wissen (Handbücher Sprachwissen 1), 335-357. Berlin, Boston: De Gruyter.

Byron, Paul, Kath Albury \& Clifton Evers (2013): „It would be weird to have that on Facebook”: young people's use of social media and the risk of sharing sexual health information. Reproductive health matters 21 (41), 35-44.

Darcy, Alison M., Barbara Dooley (2007): A clinical profile of participants in an online support group. European Eating Disorders Review 15 (3), 185-195.

Diaz, Joseph A., Rebecca A. Griffith, James J. Ng, Steven E. Reinert, Peter D. Friedmann \& Anne W. Moulton (2002): Patients' use of the Internet for medical information. Journal of General Internal Medicine 17 (3), 180-185.

Dums, Bettina (2021): Wissenstransfer im Hypertext. Sprachwissenschaftliche Analysen zum Online-Diskussionsforum des Bundesverbandes Prostatakrebs Selbsthilfe e. V. Unveröffentlichte Masterarbeit an der Universität Regensburg.

Dums, Bettina (2020): ,Neu hier, Bitte um Informationen‘. Fachsprachliche Kurzwörter und Wissenstransfer auf dem Prostata-Krebs-Diskussionsforum des Bundesverbandes Prostatakrebs-Selbsthilfe e.V. Unveröffentlichte Modularbeit an der Universität Regensburg.

Eysenbach, Gunter u. a. (2004): Health related virtual communities and electronic support groups. Systematic review of the effects of online peer to peer interactions. British Medical Journal 328 (7449), 1166.

Fleischer, Wolfgang \& Irmhild Barz (2012): Wortbildung der deutschen Gegenwartssprache. 4., völlig neu bearb. Aufl. Berlin, Boston: De Gruyter. 
Flora, Katerina, Antonios Raftopoulos \& Theodote Pontikes (2010): A Look at the Evolution of the Self-Help Movement. Journal of Groups in Addiction and Recovery 5 (3), 214-225.

Greule, Albrecht (2007): Kurzwörter in historischer Sicht. In Jochen A. Bär, Thorsten Roelcke \& Anja Steinhauer (Hrsg.), Sprachliche Kürze. Konzeptuelle, strukturelle und pragmatische Aspekte, 118-130. Berlin, New York: De Gruyter.

Hausendorf, Heiko (2009): Kleine Texte. Über Randerscheinungen von Textualität. Germanistik in der Schweiz - Onlinezeitschrift der Schweizerischen Akademischen Gesellschaft für Germanistik 6, 5-19.

Janich, Nina (2015): Kurze Texte und Kurztexte - transtextuell vernetzt?. In Mariann SkogSödersved, Ewald Reuter \& Christian Rink (Hrsg.), Kurze Texte und Intertextualität. Ausgewählte Beiträge der GeFoText-Konferenz vom 26.9. in Vaasa, 27-48. Frankfurt a. M.: Peter Lang.

Johnson, Grace J. \& Paul J. Ambrose (2006): Neo-tribes: The power and potential of online communities in health care. Communications of the ACM 49 (1), 107-113.

Kayany, Joseph M. (2004): Internet Etiquette (Netiquette). In Hossein Bidgoli (Hrsg.), The Internet Encyclopedia Volume 2 (G-O), 274-285. Hoboken: Wiley.

Kleinke, Sonja (2015): Internetforen: Laiendiskurs Gesundheit. In Albert Busch \& Thomas Spranz-Fogasy (Hrsg.), Handbuch Sprache in der Medizin (Handbücher Sprachwissen 11), 405-422. Berlin, Bosten: De Gruyter.

Kobler-Trill, Dorothea (1994): Das Kurzwort im Deutschen. Eine Untersuchung zu Definition, Typologie und Entwicklung (Germanistische Linguistik, 149). Tübingen: Niemeyer (Reprint De Gruyter 2013).

Leyhausen, Katja (2007): Kurze Texte: eine theoretische Einordnung. In Jochen A. Bär, Thorsten Roelcke \& Anja Steinhauer (Hrsg.), Sprachliche Kürze. Konzeptuelle, strukturelle und pragmatische Aspekte, 339-364. Berlin, New York: De Gruyter.

Link, Elena (2019): Gesundheitskommunikation mittels Gesundheitsportalen und OnlineCommunities. In Constanze Rossmann \& Matthias R. Hastall (Hrsg.), Handbuch der Gesundheitskommunikation. Kommunikationswissenschaftliche Perspektiven, 159-169. Wiesbaden: Springer VS.

Locher, Miriam A. \& Franziska Thurnherr (Hrsg.) (2017): Linguistik Online: Special Issue "Language and Health Online".

Lorenz, Matthias N. \& Thomas Nehrlich (2019): Kleists Anekdoten - Zur Größe der Kleinen Formen. In Andrea Allerkamp, Günter Blamberger, Anne Fleig, Barbara Gribnitz, Hannah Lotte Lund \& Martin Roussel (Hrsg.), Kleist-Jahrbuch 2019, 231-235. Stuttgart: Metzler.

Misoch, Sabina (2006): Online-Kommunikation (UTB 2835). Konstanz: UVK.

Moorhead, S. Anne, Diane E. Hazlett, Laura Harrison, Jennifer K. Carroll, Anthea Irwin \& Ciska Hoving (2013): A new dimension of health care: systematic review of the uses, benefits, and limitations of social media for health communication. Journal of medical Internet research 15 (4), e85; doi: 10.2196/jmir.1933 (17 Seiten).

Nickel, Stefan, Katharina Bremer, Marie-Luise Dierks, Marius Haack, Silke Schwinn, Bernhard Borgetto \& Christopher Kofahl (2020): Digitalisierung in der gesundheitlichen Selbsthilfe. Ergebnisse einer Online-Umfrage bei Selbsthilfeorganisationen. In Deutsche Arbeitsgemeinschaft Selbsthilfegruppen e.V., Selbsthilfegruppenjahrbuch 2020, 142-153. Gießen. https://www.uke.de/extern/ dish/ergebnisse.html (letzter Zugriff 10.11.2020).

Nikula, Henrik (2015): „Ist ein Abstract kürzer als sein Bezugstext? Zum Begriff der Kürze“. In Mariann Skog-Södersved, Ewald Reuter \& Christian Rink (Hrsg.), Kurze Texte und 
Intertextualität. Ausgewählte Beiträge der GeFoText-Konferenz vom 26.9. in Vaasa, 63-75. Frankfurt a. M.: Peter Lang.

Pappert, Steffen \& Kersten Sven Roth (Hrsg.) (2021): Kleine Texte. Frankfurt a. M.: Peter Lang. Potts, Henry W. W. (2005): Online support groups. An overlooked resource for patients. He@lth Information on the Internet 44 (1), 6-8.

Quinn, Edel M., Mark A. Corrigan, Seamus M. McHugh, David Murphy, John O' Mullane, Arnold D. Hill \& Henry Paul Redmond (2013): Who`s talking about breast cancer? Analysis of daily breast cancer posts on the internet. Breast 22 (1), 24-27.

Reimann, Sandra (im Druck): Form braucht Bedeutung: Zum Wissenstransfer fachlicher Kurzwörter durch und für medizinische Laien am Beispiel der Plattform ProstatakrebsSelbsthilfe e.V. In Lars Bülow, Günter Koch, Ulrike Krieg-Holz \& Igor Trost (Hrsg.), Remotivierung in der Sprache - Auf der Suche nach Form und Bedeutung (Linguistik in Empirie und Theorie). Heidelberg: Springer-Verlag/Metzler (Springer-Nature).

Reimann, Sandra (2020): Stress - nicht nur im Studium. Sprachwissenschaftliche Untersuchungen zur Selbsthilfe-Kommunikation im Internet als Problemlösungsstrategie und Beitrag zu „E-Mental Health“. In Matthias Johannes Bauer \& Thomas Seppelfricke (Hrsg.), Stress im Studium. Stressempfinden und Stressbewältigung bei Studierenden, 207-226. München: Utz.

Reimann, Sandra (2019): „Und hab total den Heulanfall bekommen“. Emotionskulturen im Netz am Beispiel der Selbsthilfeplattform www.hungrig-online.de. In Stefan Hauser, Martin Luginbühl \& Susanne Tienken (Hrsg.), Mediale Emotionskulturen (Sprache Kommunikation - Medien 12), 201-218. Frankfurt a. M.: Peter Lang.

Reimann, Sandra (2018): Sprache des Hungerns. Selbstreflexion, Diagnostik und sprachwissenschaftliche Untersuchungen der Internetplattform www.hungrig-online.de. Tübingen: Narr.

Reimann, Sandra (2012): „Experten“ unter sich - Besonderheiten des Sprachgebrauchs im Selbsthilfeforum hungrig-online.de. In Christian Braun (Hrsg.), Sprache und Geheimnis. Sondersprachenforschung im Spannungsfeld zwischen Arkanem und Profanem (Lingua Historica. Studien und Quellen zur Geschichte der deutschen Sprache und Literatur 4), 141-158. Berlin: Akademie.

Roelcke, Thorsten (2016): Ärzte, Pfleger und Patienten: Zur Typologie deutscher Fachkommunikation in einer mehrsprachigen Gesellschaft. Symbolae Cassovienses. Kaschauer Beiträge zur Sprache und Kultur. Košické listy o jazyku a kultúre $1,107-126$.

Rossmann, Constanze \& Paula Stehr (2019): Gesundheitskommunikation im Internet. Erscheinungsformen, Potenziale, Grenzen. In Wolfgang Schweiger \& Klaus Beck (Hrsg.), Handbuch Online-Kommunikation, 393-419. 2., vollst. überarb. Aufl. Wiesbaden: Springer VS.

Schuldt, Janina (1998): Sorten fachbezogener Vermittlungstexte IV: Beipackzettel. In Lothar Hoffmann, Hartwig Kalverkämper \& Herbert Ernst Wiegand (Hrsg.), Fachsprachen (Handbücher zur Sprach- und Kommunikationswissenschaft 14,1), 583-587. Berlin, New York: De Gruyter.

Sharf, Barbara F. (1999): Beyond netiquette. The ethics of doing naturalistic discourse research on the internet. In Steve Jones (Hrsg.), Doing Internet research: Critical issues and methods for examining the Net, 243-256. Thousand Oakes: Sage.

Siever, Torsten (2011): Texte i. d. Enge. Sprachökonomische Reduktion in stark raumbegrenzten Textsorten. Frankfurt a. M.: Peter Lang. 
Slivová, Jozefína \& Tomáš Kozík (2014): Netiquette in electronic communication. International Journal of Engineering Pedagogy (iJEP) 4 (3), 67-70.

Storrer, Angelika \& Sandra Waldenberger (1998): Zwischen Grice und Knigge: Die Netiketten im Internet. In Hans Strohner, Lorenz Sichelschmidt \& Martina Hielscher (Hrsg.), Medium Sprache (Forum Angewandte Linguistik 34), 63-77. Frankfurt a. M.: Peter Lang.

Strawbridge, Matthew (2006): Netiquette: Internet etiquette in the age of the blog. Cambridgeshire: Software Reference.

Tienken, Susanne (2013): Sharing. Zum Teilen von Erzählungen in Onlineforen. In Laura Álvarez López, Charlotta Seiler Brylla \& Philip Shaw (Hrsg.), Computer-Media ted Discourse across Languages, 17-43. Stockholm: Acta Universitatis Stockholmiensis.

Thielscher, Christian \& Britta Schulte-Sutrum (2016): Die Entwicklung der Arzt-PatientenBeziehung in Deutschland in den letzten Jahren aus Sicht von Vertretern der Ärztekammern und der Kassenärztlichen Vereinigungen. Das Gesundheitswesen 78, 8-13.

Thim-Mabrey, Christiane \& Paul Rössler (Hrsg.) (2020): Verständliches Erklären und Instruieren: Sprachwissenschaftliche Untersuchungen zu Beispielen medialer, fachlicher, behördlicher und betrieblicher Kommunikation. Regensburg: Universitätsbibliothek Regensburg.

Weinreich, Cornelia (2015): Fachinterne und fachexterne Textsorten in der Medizin. In Albert Busch \& Thomas Spranz-Fogasy (Hrsg.), Handbuch Sprache in der Medizin (Handbücher Sprachwissen 11), 389-404. Berlin, Bosten: De Gruyter.

Wiese, Ingrid (2002): Bereich Medizin: Fachsprache und Wissenstransfer. Der Deutschunterricht 54 (5), 34-45.

\section{Weitere Online-Quellen}

AWMF online - Das Portal der wissenschaftlichen Medizin: https://www.awmf.org/leitlinien. html (letzter Zugriff am 27.03.2021).

BPS: Bundesverband Prostatakrebs Selbsthilfe e.V. (2021): Forumsregeln. https://prostata krebs-bps.de/forum/forumsregeln/ (letzter Zugriff 15.07.2021).

BPS: Bundesverband Prostatakrebs Selbsthilfe e.V. (2020a): Webseite. https://www.prostata krebs-bps.de/ (letzter Zugriff 02.08.2021).

BPS \& KISP (Bundesverband Prostatakrebs Selbsthilfe e.V. \& Kontakt-, Informations- und Selbsthilfestelle zum Prostatakrebs) (2020b): Prostatakrebs-Diskussionsforum. https:// forum.prostatakrebs-bps.de/ (letzter Zugriff 02.08.2021).

Caritasverband für die Diözese Regensburg e.V.: Gesundheit. Prostatakrebs! Früh erkannt Gefahr gebannt! https://www.caritas-regensburg.de/aktuelles/presse/prostatakrebsfrueh-erkannt-gefahr-geba (letzter Zugriff 27.03.2021).

Damm, Ralf-Rainer (2021): Prostatakrebs-bezogene Abkürzungen und Fachausdrücke Deutsch und Englisch (PDF). Prostatakrebs-Diskussionsforum (Webseite). http://prostatakrebse. de/informationen/pdf/abkuerzungen.pdf (letzter Zugriff 24.07.2021).

Damm, Ralf-Rainer (2020): Bei mir wurde Prostatakrebs festgestellt - was nun? Ein Nachschlagwerk zum Prostatakrebs für von dieser Erkrankung Betroffene und ihre Angehörigen sowie ein erster (aber auch zweiter und dritter) Rat nach der Krebsdiagnose (PDF). Prostatakrebs-Diskussionsforum (Webseite). www.prostatakrebse.de/informatio nen/pdf/Erster\%20Rat.pdf (letzter Zugriff 30.04.2020). 
DKG (Deutsche Krebsgesellschaft). https://www.krebsgesellschaft.de/ (letzter Zugriff 01.09.2020).

EmAnJoTo (2018): Neu hier, Bitte um Informationen (Thread). Prostatakrebs-Diskussionsforum (Webseite). https://forum.prostatakrebs-bps.de/showthread.php?10690-Neu-hier-Bitteum-Informationen (letzter Zugriff 21.04.2020).

European Association of Urology: https://uroweb.org/guideline/prostate-cancer/ (letzter Zugriff 27.03.2021).

Fritzen, Florentine (2015): Google dir deine Krankheit. Internet als Arzt. Frankfurter Allgemeine Zeitung (FAZ.NET). http://www.faz.net/aktuell/gesellschaft/gesundheit/immer-mehrkranke-suchen-rat-in-internet-13850242.html (letzter Zugriff 23.06.2017).

Gala (2007): Hape Kerkeling „Ich habe Rücken!“ https://www.gala.de/stars/news/hape-kerke ling-ich-habe-ruecken-20006850.html (letzter Zugriff 01.10.2020).

Leitlinienprogramm Onkologie (2018): Interdisziplinäre Leitlinie der Qualität S3 zur Früherkennung, Diagnose und Therapie der verschiedenen Stadien des Prostatakarzinoms Version 5.0. https://www.leitlinienprogramm-onkologie.de/filead $\mathrm{min} /$ user_upload/Downloads/Leitlinien/Prostata_5_0/LL_Prostata_Langversion_5.0.pdf (letzter Zugriff 14.04.2020).

Leitlinienprogramm Onkologie (2015): Früherkennung Prostatakrebs. https://www.leitlinien programm-onkologie.de/patientenleitlinien/prostatakrebs/ (letzter Zugriff 27.03.2021).

Onmeda.de (2020): Männergesundheit \& Urologie. Online-Diskussion (Thread). https://www. onmeda.de/forum/m\%C3\%A4nnergesundheit (letzter Zugriff 10.11.2020).

Personalfitness (2017): Rückenschmerzen. „Ich hab Rücken!“. https://www.personalfitness. de/lifestyle/292 (letzter Zugriff 01.10.2020).

Prostata Hilfe Deutschland: https://www.prostata-hilfe-deutschland.de/prostata-news/multi parametrische-mrt-mpmrt (letzter Zugriff 27.03.2021).

Pschyrembel Online: https://www.pschyrembel.de/ (letzter Zugriff 01.10.2020).

Radiopaedia (2020): Prostate Imaging-Reporting and Data System (PI-RADS). https://radiopae dia.org/articles/prostate-imaging-reporting-and-data-system-pi-rads-1 (letzter Zugriff 1.10.2020).

Takeda Pharma Vertriebs GmbH \& Co. KG: prostata.de (letzter Zugriff 08.07.2020).

Williams, Ray (2019): The Problem With the Self-Help Movement: https://raywilliams.ca/prob lem-self-help-movement/ (letzter Zugriff 16.07.2021). 